\title{
Análisis de la Fidelización del Estudiante de Ingeniería con su Centro de Educación Superior: Desafíos de Gestión Educacional
}

\author{
Segundo R. Cabana, Felicindo H. Cortés, Domingo L. Vega y Rodrigo A. Cortés \\ Universidad de La Serena, Fac. de Ingeniería, Dpto. de Ing. Industrial, Casilla 554, La Serena-Chile \\ (e-mail: rcabana@userena.cl, fcortes@userena.cl, dvega@userena.cl, \\ rcortes.investigacion.dii.uls@gmail.com)
}

Recibido Abr. 26, 2016; Aceptado Jun. 16, 2016; Versión final Jul. 15, 2016, Publicado Dic. 2016

\begin{abstract}
Resumen
El objetivo de la investigación es realizar un análisis de la fidelización del estudiante de ingeniería en relación con su centro de educación superior. Se toma como población objetivo los estudiantes de la carrera Ingeniería Civil Industrial de la Universidad de La Serena, en Chile y se propone un modelo relacional con seis hipótesis de trabajo. Se aplicaron encuestas con tamaño de muestra de 177 estudiantes que representan el $54 \%$ de la población, considerando escala politómicas tipo Likert. Para el contraste del modelo propuesto se emplearon los métodos de ecuaciones estructurales. Finalmente, se determinó la confirmación del carácter multidimensional de la fidelidad del estudiante con su centro de educación superior. Basado en ello, se concluye que la fidelidad del estudiante es afectada de forma significativa por la calidad del proceso enseñanza-aprendizaje, que la satisfacción del estudiante influye de manera positiva y significativa, y que la red de apoyo externo es una variable significativa que impacta directa e indirectamente la fidelidad del estudiante.
\end{abstract}

Palabras clave: estudiante, enseñanza-aprendizaje, satisfacción, fidelización, red de apoyo

\section{Analysis of Engineering Student Loyalty to his/her Center of Higher Education: Education Management Challenges}

\begin{abstract}
The objective of the research is to analyze the loyalty of engineering students in relation to its center of higher education. Students of the career of Industrial Civil Engineering at the University of La Serena in Chile were considered in the study and a model including six relational hypotheses is proposed. Surveys were applied to 177 students, representing $54 \%$ of the population, considering Likert scale polytomous. To compared the results of the proposed model structural equation methods were used. Finally, confirmation of the multidimensional nature of student loyalty with its center of higher education was determined. Based on this, it is concluded that loyalty student is significantly affected by the quality of the teaching-learning process, student satisfaction has a positive and meaningful influence, and that the network of external support is a significant variable that impacts directly and indirectly students 'loyalty.
\end{abstract}

Keywords: student, teaching and learning, satisfaction, loyalty, support network 


\section{INTRODUCCIÓN}

En los últimos años las demandas de la sociedad se han expresado intensamente respecto a una Reforma Educacional, que establece las siguientes prioridades en la educación superior: gratuidad, acreditación, organización institucional y rol de las universidades estatales e incremento de los recursos para investigación en ciencia y tecnología (Bitar, 2015). Los desafíos estratégicos que crecientemente se están generando, se justifican en parte pues aun siendo muy generosos en la evaluación del actual sistema de educación superior de Chile, se debe concluir que éste es deficiente, que está en crisis y que no responde a los requerimientos más esenciales de una sociedad en la era del conocimiento (Rodríguez, 2012). En ese contexto el aseguramiento de la calidad es un imperativo estratégico en la educación universitaria (Pedraja y Rodríguez, 2015), por lo que la innovación en gestión y marketing educacional tendrá un desarrollo exponencial, donde la satisfacción de los estudiantes es un resultado importante porque está asociada a un mayor involucramiento en el proceso y una mayor motivación para el aprendizaje (Negrao et al., 2014).

El proceso de enseñanza-aprendizaje es, fundamentalmente, un proceso de "encuentro humano" en el que tiene lugar el diálogo atento, inteligente y razonable, así como la libre valoración sobre los diversos aspectos de la realidad que se estudia, con el propósito de ampliar el "horizonte de comprensiones, significados y valores que intervienen en él" (Patiño, 2012). El aumento de las expectativas de la sociedad en lo que concierne a la labor de universidades está generando una mayor priorización para mejorar la calidad de la enseñanza, investigación y todos los servicios que una universidad proporciona. Este interés ha conducido a dar un énfasis mayor sobre el análisis de dos aspectos que se encuentran estrechamente ligados: calidad y satisfacción (de la Fuente et al., 2010). La satisfacción del estudiante refleja la eficiencia de los servicios académicos y administrativos y es importante saber que los estudiantes manifiesten su satisfacción con las unidades de aprendizaje, con las interacciones con su profesor y compañeros de clase, así como con las instalaciones y el equipamiento (Jiménez et al., 2011). Respecto de la calidad, los académicos le deben asignar una relevancia prioritaria a sus actividades docentes dentro del total de sus obligaciones académicas (Asún et al., 2013), pues uno de los indicadores más importantes para medir la calidad de la enseñanza tiene que ver con el grado de satisfacción de las personas involucradas en el proceso educativo (Jiménez et al., 2011).

Además, el mejoramiento continuo de la calidad del proceso enseñanza-aprendizaje, requiere que la visión del estudiante, producto de sus percepciones, expectativas y necesidades, sirva como indicador para el mejoramiento de la gestión y el desarrollo de los programas académicos (Álvarez et al., 2014). Así mismo, es necesario apuntar que la satisfacción del estudiante es el eje central de todos los procesos que se llevan a cabo en las universidades pues su principal función sustantiva es la docencia centrada en él (Salinas et al., 2008). En el marco de este pensamiento se ha establecido que la calidad de las instituciones de educación depende, cada vez más, de la retroalimentación que estas reciban de sus estudiantes (Suárez, 2012). Es necesario que la docencia esté acompañado de servicios adicionales y reputación de la universidad, capaces de incrementar la satisfacción global del estudiante al incrementar el valor del servicio ofertado (de la Fuente et al., 2010). Un área de oportunidad para incrementar la calidad Universitaria y por tanto la satisfacción del estudiante es promover proyectos para mejorar la calidad de los servicios administrativos ofrecidos, además de ser indispensable mejorar las condiciones de Infraestructura, principalmente de las Instalaciones deportivas y sanitarias (Álvarez et al., 2014).

En este contexto el estudio que se presenta se basa en seis hipótesis relacionadas con los conceptos de satisfacción y fidelidad. Hipótesis $1(\mathrm{H} 1)$ : la Calidad del proceso enseñanza-aprendizaje influye directa y positivamente sobre la satisfacción del estudiante; Hipótesis $2(\mathrm{H} 2)$ : la Satisfacción influye directa y positivamente sobre la fidelidad del estudiante; Hipótesis $3(\mathrm{H} 3)$ : la red de apoyo externo influye directa y positivamente sobre la satisfacción del estudiante; Hipótesis $4(\mathrm{H} 4)$ : la red de apoyo externo influye directa y positivamente en la fidelidad del estudiante con la Universidad; Hipótesis 5 (H5): la red de apoyo externo influye directa y positivamente sobre la calidad del proceso enseñanza-aprendizaje en pre-grado; e Hipótesis $6(\mathrm{H} 6)$ : la influencia de la calidad proceso enseñanza-aprendizaje influye directa y positivamente en la fidelidad del estudiante

\section{SATISFACCIÓN Y FIDELIDAD}

La satisfacción es la apreciación favorable que hacen los estudiantes de los resultados y experiencias asociadas con su educación, en función de la atención a sus propias necesidades y al logro de sus expectativas (Candelas et al., 2013). Se considera satisfacción al nivel del estado de ánimo de una persona que resulta de comparar el rendimiento percibido de un producto o servicio con sus expectativas (Gómez et al., 2013). Su relevancia se debe básicamente a su carácter antecesor de la fidelidad del cliente y de la intención de éstos de hablar positivamente de la organización (de la Fuente et al., 2010). 
La teoría de la gestión de la calidad total instala la idea del estudiante como cliente de las instituciones educativas, esto implica que de haber quedado satisfecho con el servicio y/o producto recibido buscaría repetir la experiencia y se mantendría fiel a "la marca" de la institución y carrera. Pero, como la educación no se consume de la misma manera que otros bienes, lo que se espera del estudiante fidelizado es que recomiende a sus parientes, conocidos y amigos que repitan su experiencia (Suárez, 2012), creando o mejorando la imagen de la organización, actuando como "captadores" de nuevos estudiantes y asegurando hasta cierto punto su supervivencia (de la Fuente et al., 2010). Lo cual implica un cierto nivel de continuidad en la relación del cliente-estudiante y en la forma en la que se da esa relación con el Centro de Educación Superior-CES (Schlesinger et al., 2014). Por supuesto, no existe un consenso en asignar al estudiante el rol de cliente, dada la diversidad de tareas asignados a la universidad (de la Fuente et al., 2010).

\section{Red de apoyo externo y satisfacción del estudiante}

Los estudiantes universitarios tienen estructuras de apoyo interno y externo, en el ámbito interno sus compañeros de carrera, amigos, familiares y en el ámbito externo los empleadores o empresarios (Cabrera et al., 2006). Tanto los aspectos académicos como los sociales son dimensiones influyentes en la satisfacción de los estudiantes (Blázquez et al., 2013). El apoyo social percibido es un constructo multifacético conceptualizado como la valoración cognitiva de que existe una relación de confianza con los otros, con los que se puede contar en caso de necesidad. De este modo se entienden las redes como formas de interacción social, espacios de convivencia y conectividad. (Bernasconi, 2009).

Estudios recientes sobre los determinantes del logro académico de los universitarios destacan el apoyo social percibido. Su efecto deriva del sentimiento de mutua confianza hacia otras personas con las que se puede contar en caso de necesidad; esto mediatiza la valoración sobre las circunstancias problemáticas o estresantes y la apreciación de los recursos de que se dispone para afrontarlas (Martínez et al., 2014). El entorno familiar ocupa un lugar importante, pues afecta el desarrollo del individuo, manifestándose también en la vida académica. Si bien el grupo de iguales y los profesores adquieren una relevancia creciente, ello no significa que la familia pierda importancia, sino que comparte con aquellos su papel en el enriquecimiento y desarrollo del autoconcepto académico (Santana y Feliciano, 2011).

\section{Red de apoyo externo y la fidelidad del estudiante}

La red de apoyo, permite a la educación ampliar el tejido social y las fronteras de sus construcciones simbólicas y significativas de los actores educativos a partir de la constatación de sus vínculos interpersonales y socio-profesionales. Igualmente importante son los procesos afectivos o emocionales implicados en el aprendizaje, la formación de una relación con los estudiantes, y que cuidan sobre el aprendizaje y la capacidad de integrar y aplicar la nueva información de los estudiantes (Lilian, 2014).

Además de los vínculos con las estructuras y organizaciones que conforman el medio ambiente más inmediato, la universidad también mantiene relación con las organizaciones, organismos y otras instituciones de la localidad para la planificación, organización y desarrollo del proceso pedagógico. La universidad, como sistema abierto, es profundamente influida por el entorno. Toda la actividad de ella y de las propias organizaciones, organismos e instituciones está condicionada por la permeabilidad o apertura al medio ambiente próximo y mediato, lo que exige establecer mecanismos bidireccionales para abrirse a sus influencias (Bertoglia, 2005).La diferencia con esta manera de asumir los cambios del mercado laboral por parte de los estudiantes, radica en investigar las exigencias del entorno, y de las organizaciones o los trabajos a los que pretenden vincularse, para llevar a cabo acciones que les permitan acercarse a lo requerido, y tener mayores posibilidades de ser empleables (Renteria y Andrade, 2007), lo que directamente influye en la fidelidad del estudiante. El propósito es reducir las percepciones de estudiantes y potenciales empleadores, que se quejan del distanciamiento cada vez mayor existente entre la formación universitaria y las necesidades reales de las empresas y del exigente mercado laboral (del Val Merino, 2013).

La propuesta de formación de redes de apoyo tendrá éxito siempre y cuando las instituciones formen y consoliden sus vínculos y compartan ambientes de aprendizaje abierto y a distancia, donde la tecnología educativa sea aprovechada para impulsar los procesos de enseñanza-aprendizaje y donde cada vez más se desarrolle en el estudiante el estudio independiente, uso de la tecnología y trabajo colaborativo, sólo así las redes de apoyo tendrán éxito y con ello, podrá reducirse la desigualdad social y la inequidad educativa de la que tanto se habla (Barroso y Colón, 2009), mejorando la satisfacción por el servicio y la fidelidad con la institución.

\section{Red de apoyo externo y calidad del proceso enseñanza-aprendizaje}

La calidad de las instituciones de educación depende, cada vez más, de la retroalimentación que éstas reciban de sus egresados, por ello, realizar encuestas de opinión debe ser prioridad en este tipo de 
organizaciones (Suárez, 2012), lo que implica considerar el entorno para mejorar los procesos de enseñanza-aprendizaje. Esto permitirá identificar los principales aspectos y desafíos que deben superar las Universidades para que los estudiantes estén satisfechos con los servicios y productos que ofrecen. Integrar a la red externa para mejorar el valor del servicio educacional, cada vez es más factible empleando las TIC (Tecnologías de la Información y Comunicación), las cuales se transforman en un elemento innovador de ayuda a la docencia, ya que permiten un cambio de mentalidad o paradigma en el docente, centrando el foco de enseñanza en el estudiante (Avagliano y Vega, 2013; López et al., 2012).

La interacción de sus egresados, potenciales empleadores e instituciones relacionadas con la carrera del estudiante, al menos representa una "tutoría", que es un recurso muy importante en la formación inicial y continua del alumnado, porque permite un acercamiento a un proceso educativo individualizado que facilita espacios y tiempos que no siempre se encuentran en la clase ordinaria y que son imprescindibles para mejorar la calidad de la enseñanza (Cantero y Mato, 2014). El hecho de visualizar diferentes espacios y experiencias con colegas de profesión proporciona un nuevo sentido a los estudiantes, son factores que pueden determinar una mejor participación y la aceptación por gran parte de los estudiantes, lo que influye en la calidad del proceso de enseñanza-aprendizaje, pues el estudiante incorpora habilidades que demandan las empresas (Aguiar et al., 2010), pues claramente fortalecer el vínculo del estudiante con su red externa y particularmente con su potencial mercado laboral, adquiere cada más relevancia pues el empleo se ha convertido en los últimos años en un tema controvertido que genera cierta sensación de frustración entre los jóvenes titulados (Conchado y Carot, 2013).

Por consiguiente, para que exista una mejor calidad educativa, es necesario que los clientes externos dialoguen con los clientes internos y estos últimos, a su vez, deben prepararse para participar en la mejora continua del proceso educativo, de forma que puedan entender las necesidades mutuas (Saraiva, 2008). Así entonces, la Universidad empuja la construcción de nuevo conocimiento en su estructura tradicional de pasar o reinterpretar el conocimiento preexistente a una actividad complementaria no sin pocos contradictores, en la cual la universidad aprovecha procesos de interacción externa como un mecanismo de búsqueda de nuevos problemas de conocimiento o búsqueda de respuestas a nuevos necesidades del entorno (Montoya y Aguilar, 2013).

\section{Calidad proceso enseñanza-aprendizaje y fidelización del estudiante}

Las instituciones de educación superior, como cualquier otra organización de servicio, ha de estar orientada a mantener y mejorar continuamente la satisfacción de los servicios a los clientes, contribuyendo a la mejora de los sistemas educativos, a la evolución de la universidad en sus procesos y, en general, para hacer posible su progreso (Agustín y Domelis, 2009). Por supuesto, estos desafíos se superan de manera sostenible si participa el estudiante de manera activa, para lo cual se requiere que asuma un rol de cliente que precisan de ser satisfechos (Candelas et al., 2013) y fidelizados (Manzano, 2010). Dicha evolución se debe en parte a la modificación del papel del alumnado, ya que ahora el aprendizaje no solo está centrado en la adquisición de conocimientos, sino que el estudiante también debe actuar como motor de su aprendizaje ante cualquier situación o experiencia educativa a lo largo de toda su vida (Cantero y Mato, 2014). El vínculo que genera el estudiante de pregrado con la universidad, se relaciona con la capacidad que tiene éste de escoger seguir con sus estudios en la institución universitaria en la que se encuentra y no de cambiarse a otra que le genere expectativas distintas. Es por ello que, las universidades deben crear lazos de fidelidad o al menos retención del alumnado (Donoso y Schiefelbein, 2007). Así entonces los procesos de fidelización del estudiante, son consecuencia de la satisfacción y la calidad percibida por los servicios educacionales consumidos, que en este caso depende de la calidad del proceso de enseñanzaaprendizaje (Pereira, 2011).

Considerando todas las relaciones anteriores, se propone un modelo causal que busca identificar las variables que influyen en la formación de un vínculo relacional entre los alumnos de ingeniería y su Universidad (Fig. 1).

\section{METODOLOGÍA}

Con el objeto de validar empíricamente el modelo propuesto, se presenta un estudio realizado sobre una muestra de 177 estudiantes de la Carrera de Ingeniería Civil Industrial (ICI), de la Universidad de La Serena (ULS) - Chile. Esta muestra representa el $54 \%$ de los estudiantes de la carrera, misma que participa con el $11 \%$ de los estudiantes a nivel de la Facultad de Ingeniería y que poseen un perfil de ingreso (socioeconómico y académico) equivalente a los otros estudiantes de las diferentes carreras de la Facultad. El trabajo de campo fue realizado mediante una encuesta personal durante el período de clases, para lo cual se generaron las mejores condiciones y apoyos necesarios para reducir sesgos y confusiones. La Ficha Técnica del proceso de muestreo se presenta en la tabla 1. 


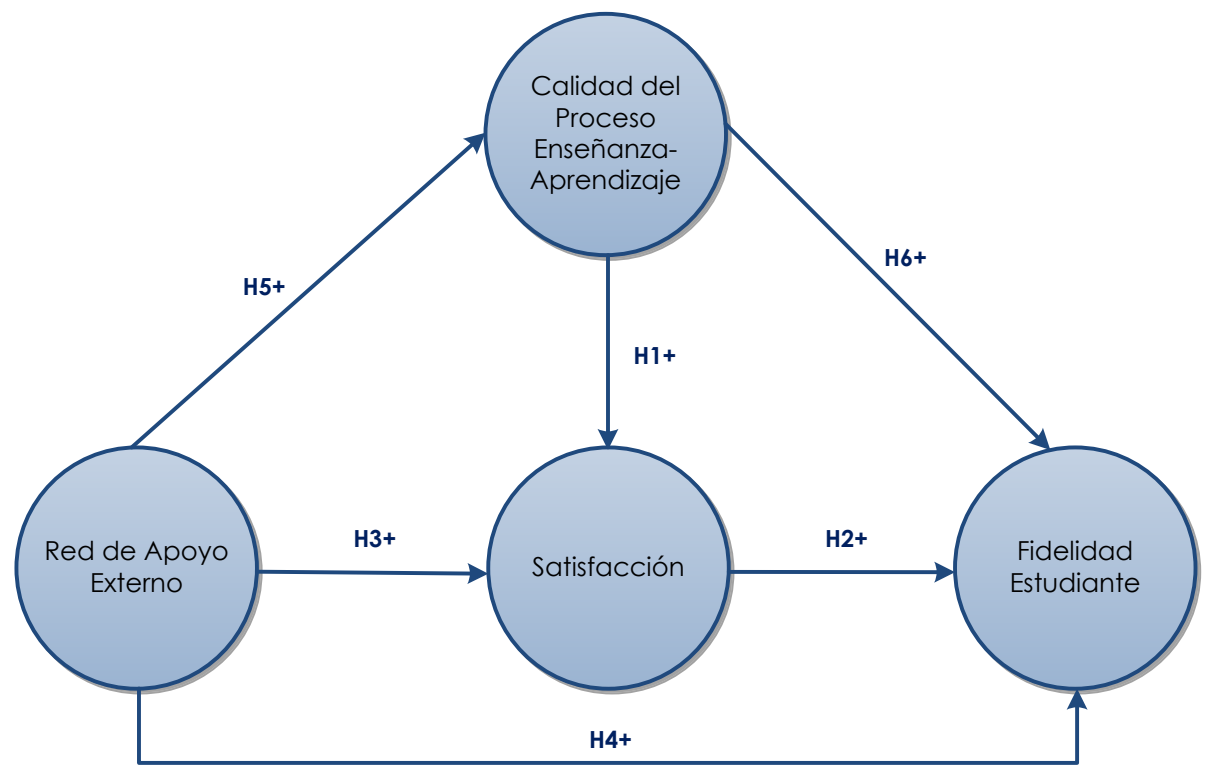

Fig. 1: Modelo causal sobre la Fidelidad del estudiante en su Centro de Educación Superior

Los resultados revelan que la muestra se integra por $50,3 \%$ de hombres y $49,7 \%$ de mujeres, la edad predominante de la muestra se encuentra en los 23 años $(20,9 \%)$, siendo el ingreso familiar más habitual entre los US\$ 360 y US\$1079,7 al mes en un 43,8\% de los casos. En tanto que el tipo de educación más predominante que recibieron los encuestados en la enseñanza media, corresponde a la educación subvencionada $(71,8 \%)$, mientras que el $79,7 \%$ de los estudiantes de Ingeniería Civil Industrial viven con sus familias (Ver Tabla 2).

Tabla 1: Ficha técnica de la investigación

\begin{tabular}{|l|l|}
\hline \multicolumn{2}{|c|}{ Ficha Técnica de la Investigación } \\
\hline Tipo de muestreo & Muestreo probabilístico \\
\hline Nivel de confianza & $95 \% ; \mathrm{z}=1,96 ; \mathrm{p}=\mathrm{q}=0,5$ (5\% error y 95\% de confiabilidad). \\
\hline Tamaño muestral & 177 Estudiantes \\
\hline Unidad de análisis & Carrera de Ingeniería Civil Industrial (ICl), Universidad de La Serena \\
\hline Método de recogida de la información & Cuestionario presencial de 34 afirmaciones \\
\hline Tipo de pregunta encuesta & $\begin{array}{l}\text { Politómicas tipo Likert (1 a } 5) \text { puntuaciones o categorías y de } \\
\text { selección }\end{array}$ \\
\hline Fecha de realización del trabajo de campo & Entre Junio y Julio de 2014 \\
\hline
\end{tabular}

\section{Fiabilidad individual de los indicadores}

Para determinar la fiabilidad individual de los indicadores se calcularon las cargas factoriales de cada uno, lo que es aceptable pues el índice Kaiser-Meyer-Olkin (KMO) que para cada uno de los constructos fue superior a 0,50 y la prueba de esfericidad de Bartlett (PEB) debe ser significativa $(p<0,05)$, según Williams et al. (2010). Para realizar lo anterior, se utilizó el software SPSS Statistics versión 21, utilizando el método de extracción por Componentes Principales. Para aceptar un indicador como integrante de un constructo se han establecido diversos valores de cargas factoriales dependiendo del autor, para el caso de esta investigación se aceptan las cargas factoriales con valores superiores a 0,6 fijado por (Falk y Miller, 1992; Schlesinger et al., 2014). Los resultados se encuentran tabulados en tabla 3, se observa que el indicador CAE6, CAE7 y CAE8 no son aceptados al tener una carga inferior a 0,6, es decir, se eliminaron estos indicadores correspondiente al constructo "Calidad del proceso enseñanza-aprendizaje". El resto de los ítems resultaron aceptables y significativos al 95\%.

En la misma tabla, se determinó la comunalidad de las variables manifiestas que es la parte de la varianza que es explicada por el constructo (Bollen, 1989). Para el cálculo de la comunalidad se estimó el cuadrado de la correlación entre las variables manifiestas y su propia variable latente. Por ejemplo, para el primer indicador S1 se tiene una carga factorial de 0,808, que representa una comunalidad de 0,6529, lo que indica que el $65,29 \%$ de la varianza de la variable manifiesta está relacionada con el constructo satisfacción (Ver Tabla 3). En la Tabla, el símbolo $\left(^{*}\right)$ indica que $t>1,96(p<0,05)$. Todos los valores indican carga significativa al $95 \%$. Además, 1 US $\$=555,635$ CLP (Promedio entre Junio y Julio de 2014). 
Tabla 2. Datos descriptivos de la muestra. Características socio-demográficas de la muestra.

\begin{tabular}{|c|c|c|c|c|c|}
\hline Variable & Ítem & $\begin{array}{c}\% \text { en la } \\
\text { muestra }\end{array}$ & Variable & Ítem & $\begin{array}{l}\% \text { en la } \\
\text { muestra }\end{array}$ \\
\hline \multirow{2}{*}{ Sexo } & Hombre & 50,3 & \multirow{5}{*}{$\begin{array}{c}\text { Nivel } \\
\text { Socioeconómico }\end{array}$} & US $\$ 0$ - US\$ 359,9 & 9,7 \\
\hline & Mujer & 49,7 & & US $\$ 360$ - US $\$ 1079,8$ & 43,8 \\
\hline \multirow{9}{*}{ Edad } & 17 & 1,1 & & US\$1079,8 - US\$2159,7 & 33,5 \\
\hline & 18 & 15,8 & & US\$2159,7 - US\$3599,5 & 9,1 \\
\hline & 19 & 11,3 & & Más de US\$3599,5 & 9,7 \\
\hline & 20 & 18,1 & \multirow{3}{*}{$\begin{array}{l}\text { Tipo de } \\
\text { educación } \\
\text { escolar }\end{array}$} & Municipal & 20,9 \\
\hline & 21 & 11,3 & & Subvencionada & 71,8 \\
\hline & 22 & 9,6 & & Particular & 6,2 \\
\hline & 23 & 20,9 & \multirow{3}{*}{$\begin{array}{l}\text { Lugar de } \\
\text { residencia }\end{array}$} & Arrienda & 18,6 \\
\hline & 24 & 6,8 & & Vive con sus familiares & 79,7 \\
\hline & $>25$ & 5,1 & & Hogar de la ULS & 1,7 \\
\hline \multirow{2}{*}{$\begin{array}{l}\text { Profesionales } \\
\text { en la familia }\end{array}$} & Sí & 67,8 & \multirow{2}{*}{ Tiene Hijos } & Sí & 5,1 \\
\hline & No & 32,2 & & No & 94,9 \\
\hline \multirow{2}{*}{ Trabaja } & Sí & 21,5 & \multirow{5}{*}{$\begin{array}{l}\text { Vive con su } \\
\text { pareja }\end{array}$} & \multirow{3}{*}{ Sí } & \multirow{3}{*}{8,5} \\
\hline & No & 78,5 & & & \\
\hline \multirow{3}{*}{$\begin{array}{l}\text { Forma de } \\
\text { Pago }\end{array}$} & Subsidio Estatal & 60,2 & & & \\
\hline & Bancario & 9,70 & & \multirow[b]{2}{*}{ No } & \multirow[b]{2}{*}{91,5} \\
\hline & $\begin{array}{c}\text { Personal y/o } \\
\text { Familiar }\end{array}$ & $30,1 \%$ & & & \\
\hline
\end{tabular}

Tabla 3. Modelo de medición: Fidelidad del Estudiante

\begin{tabular}{|c|c|c|c|c|}
\hline Constructo & Indicador & Ítem & $\begin{array}{c}\text { Carga } \\
\text { Factorial }(\lambda)\end{array}$ & $\begin{array}{l}\text { Comunalidad } \\
\left(\lambda^{2}\right)\end{array}$ \\
\hline \multirow{6}{*}{ Satisfacción } & S1 & Aprendizaje con valor desarrollo personal & $0,808^{*}$ & 0,6529 \\
\hline & S2 & Aprendizaje con valor desarrollo profesional & $0,854^{*}$ & 0,7293 \\
\hline & S3 & Aprendizaje con valor Empleabilidad & $0,821^{*}$ & 0,6740 \\
\hline & S4 & Aprendizaje con valor Emprendimiento & $0,836^{*}$ & 0,6989 \\
\hline & S5 & Nivel de satisfacción con la carrera & $0,620^{*}$ & 0,3844 \\
\hline & S6 & Nivel de satisfacción con la universidad & $0,693^{*}$ & 0,4802 \\
\hline \multirow{4}{*}{$\begin{array}{l}\text { Fidelidad del } \\
\text { Estudiante }\end{array}$} & $\mathrm{F} 1$ & Nivel de relación con la carrera & $0,798^{*}$ & 0,6368 \\
\hline & $\mathrm{F} 2$ & Nivel de relación con la universidad & $0,811^{*}$ & 0,6577 \\
\hline & F3 & Promotor de la universidad & $0,831^{*}$ & 0,6906 \\
\hline & $\mathrm{F} 4$ & Continuará con cursos de postgrados & $0,657^{*}$ & 0,4316 \\
\hline \multirow{8}{*}{$\begin{array}{l}\text { Calidad del } \\
\text { proceso } \\
\text { enseñanza- } \\
\text { aprendizaje }\end{array}$} & CAE1 & Nivel de motivación por el proceso & $0,697^{*}$ & 0,4858 \\
\hline & CAE2 & Libertad de decisión para mejorar el proceso & $0,670^{*}$ & 0,4489 \\
\hline & CAE3 & Profesor me involucra en mi aprendizaje autónomo & $0,755^{*}$ & 0,5700 \\
\hline & CAE4 & Mejoran mis capacidades al aprender de los errores & $0,628^{*}$ & 0,3944 \\
\hline & CAE5 & Redes de apoyo interno mejoran mis capacidades & $0,630^{*}$ & 0,3969 \\
\hline & CAE6 & $\begin{array}{l}\text { El diseño del proceso E-A me genera resultados } \\
\text { académicos adecuados }\end{array}$ & $0,321^{*}$ & 0,1030 \\
\hline & CAE7 & Autonomía para influir en el proceso de E-A & & \\
\hline & CAE8 & Redes de apoyo externo mejoran mis capacidades & $0,566^{*}$ & 0,3203 \\
\hline \multirow{4}{*}{$\begin{array}{l}\text { Red de apoyo } \\
\quad \text { externa }\end{array}$} & RAE1 & Influencia en la colocación laboral o autoempleo & $0,632^{*}$ & 0,3994 \\
\hline & RAE2 & Comunicación motiva a fortalecer mis capacidades & $0,903^{*}$ & 0,8154 \\
\hline & RAE3 & Retroalimentación influye en mi autodesarrollo & $0,879^{*}$ & 0,7726 \\
\hline & RAE4 & $\begin{array}{l}\text { Motivan a actuar para mejorar colocación laboral o } \\
\text { autoempleo }\end{array}$ & $0,623^{*}$ & 0,3881 \\
\hline
\end{tabular}




\section{Fiabilidad del constructo}

Para evaluar la consistencia interna de los indicadores que miden los constructos reflectivos, se ha analizado la fiabilidad del constructo por medio del alfa de Cronbach y el coeficiente de fiabilidad compuesta (IFC). Para analizar la primera prueba de consistencia interna se ha utilizado el paquete estadístico SPSS versión 21. En la Tabla 4 se observa que la fiabilidad compuesta (IFC) como los a de Cronbach presentan una alta consistencia interna en los 4 constructos analizados, ya que ambas medidas superan los niveles recomendados de 0,7 y 0,8 respectivamente (Gefen y Straub, 2005; Fuentes y Moliner, 2014), lo que verifica por tanto la consistencia interna de las variables del instrumento de evaluación.

Tabla 4. Fiabilidad del constructo: Fidelidad del Estudiante

\begin{tabular}{|l|c|c|c|}
\hline Constructo & Alfa de Cronbach $(\alpha)$ & Índice fiabilidad compuesta (IFC) & Análisis Varianza Extraída (AVE) \\
\hline Satisfacción & 0,854 & 0,900 & 0,603 \\
\hline Fidelidad & 0,775 & 0,858 & 0,604 \\
\hline $\begin{array}{l}\text { Calidad del proceso } \\
\text { enseñanza-aprendizaje }\end{array}$ & 0,757 & 0,809 & 0,559 \\
\hline Red de apoyo externa & 0,769 & 0,850 & 0,594 \\
\hline Bondad de Ajuste & Chi-Square:1565; Df:171; $\mathrm{p}=; 0,000$ RMSEA=0,0928; NFI=0,936; IFI=0,901; CFI: 0,998 \\
\hline
\end{tabular}

Validez del constructo

Para valorar la validez del constructo se realizaron dos análisis fundamentales: la validez convergente y la validez discriminante.

La validez convergente, se comprueba a través de la varianza extraída media (AVE) de los constructos. EI coeficiente AVE proporciona la cantidad de varianza que un constructo reflectivo obtiene de sus indicadores con relación a la cantidad de varianza debida al error de medida. Como se puede observar en la Tabla 4, el coeficiente AVE para todos los valores alcanzados superan el valor mínimo recomendado de 0,5 (Vinzi et al., 2010; Chin, 1998; Fornell y Larcker, 1981) y son significativos al 95\%, por lo que se puede afirmar que las variables explican más del $50 \%$ de la varianza de sus constructos y por lo tanto existe validez convergente.

La validez discriminante, se verifica si los valores de la matriz de correlaciones entre constructos que está formada por la raíz cuadrada del coeficiente AVE es superior al resto de los valores de su misma columna (Espejel et al., 2013). Para agilizar el procedimiento, Sanguino (2006) propone un procedimiento inverso, en donde se calcula la raíz cuadrada de la AVE, que ha de ser mayor que las correlaciones que presentan con el resto de constructos, sus resultados son CAE: 0748; S: 0777; RAE: 0,771 y L: 0,777, lo que corresponde a indicadores que garantizan la validez discriminante del modelo propuesto.

\section{RESULTADOS}

Se planteó un análisis de ecuaciones estructurales para el contraste del modelo de relaciones propuesto (Figura 1). Se observa que los índices de bondad de ajuste del modelo global son muy aceptables (Tabla 5), lo que sugiere que la red nomológica de relaciones se ajusta a los datos obtenidos en el estudio. Para contrastar las hipótesis se utiliza el Método Basado en Covarianza (MBC), debido a que este método está orientado a la estimación de parámetros y trabaja con indicadores reflectivos.

\section{Análisis de relaciones causales y contraste de hipótesis}

Para determinar la pertinencia del modelo propuesto se utilizaron indicadores de bondad de ajuste, los cuales son indicadores de la pertinencia de una teoría de relaciones entre variables, dada por la correspondencia entre el modelo que la representa y los datos utilizados para probar esa teoría (Gaxiola J. et al., 2012). Los indicadores estudiados corresponden al índice de ajuste normalizado y no normalizado (BBNFI y BBNNFI), índice de ajuste comparativo (CFI), índice de ajuste incremental (IFI), índice de error medio cuadrático de aproximación (RMSEA), los cuales se detallan en la tabla 5 . Al eliminar los 3 ítems ya señalados, se observa en dicha tabla que las medidas de bondad de ajuste indican estar dentro de los niveles recomendados, por lo tanto, la calidad del ajuste tomando en consideración el contraste $\mathrm{Chi}^{2}$ Robusto de Satorra-Bentler y observando los indicadores globales planteados anteriormente, se puede afirmar que el ajuste global es aceptable. Así mismo, los resultados indican la existencia de relaciones causales significativas y positivas entregadas por el coeficiente estandarizado y por $t$-value de los constructos superiores a 1,96 en las hipótesis planteadas inicialmente, por lo que se comprueban y aceptan las seis hipótesis propuestas. 
Tabla 5. Modelo de Ecuaciones Estructurales: Análisis de relaciones causales y contraste de hipótesis.

\begin{tabular}{|c|c|c|c|c|}
\hline Hipótesis & Relación Estructural & $\begin{array}{l}\text { Coeficiente } \\
\text { Estandarizado }\end{array}$ & $\begin{array}{l}\text { Ratio Crítico } \\
\text { (t-value) }\end{array}$ & Contraste \\
\hline $\mathrm{H} 1$ & $\begin{array}{l}\text { Calidad Proceso Enseñanza-Aprendizaje } \rightarrow \\
\text { Satisfacción Estudiante }\end{array}$ & $0,75^{* *}$ & 3,397 & Acepta \\
\hline $\mathrm{H} 2$ & $\begin{array}{l}\text { Satisfacción Estudiante } \rightarrow \text { Fidelidad del } \\
\text { Estudiante }\end{array}$ & $0,80^{* *}$ & 4,360 & Acepta \\
\hline $\mathrm{H} 3$ & $\begin{array}{l}\text { Red de Apoyo Externo } \rightarrow \text { satisfacción } \\
\text { Estudiante }\end{array}$ & $0,13^{* *}$ & 2,610 & Acepta \\
\hline $\mathrm{H} 4$ & $\begin{array}{l}\text { Red de Apoyo Externo } \rightarrow \text { Fidelidad del } \\
\text { Estudiante }\end{array}$ & $0,16^{* *}$ & 2,131 & Acepta \\
\hline H5 & $\begin{array}{l}\text { Red de Apoyo Externo } \rightarrow \text { Calidad Proceso } \\
\text { Enseñanza-Aprendizaje }\end{array}$ & $0,03^{* *}$ & 2,680 & Acepta \\
\hline $\mathrm{H} 6$ & $\begin{array}{l}\text { Calidad Proceso Enseñanza-Aprendizaje } \rightarrow \\
\text { Fidelidad del Estudiante }\end{array}$ & $0,75^{\star \star}$ & 3,397 & Acepta \\
\hline & \multicolumn{4}{|l|}{ **: t-value $>1,96(p-$ value $<0,05)$} \\
\hline \multirow{2}{*}{$\begin{array}{l}\text { Bondad de } \\
\text { Ajuste }\end{array}$} & \multicolumn{4}{|c|}{ Chi-Square:419,343; Df:146; $p=; 0,000 ;$ RMSEA=0,0982; BBNFI=0,943; IFI=0,916; CFI:0,913 } \\
\hline & \multicolumn{4}{|c|}{$\begin{array}{l}\text { Como ajustes aceptables se tiene que: BBNFI > 0,9 (Mercado y Gil, 2012); CFI > 0,9 (González y } \\
\text { Backhoff, 2010); IFI tiene un valor cercano a } 1 \text { (González y Backhoff, 2010); RMSEA < 0,1 (González y } \\
\text { Backhoff, 2010). }\end{array}$} \\
\hline
\end{tabular}

\section{DISCUSIÓN}

Una primera contribución de esta investigación, es la confirmación del carácter multidimensional de la fidelidad del estudiante de pregrado con la universidad, constituidas por la Satisfacción del estudiante, la Calidad del Proceso Enseñanza-Aprendizaje y la Red de Apoyo Externo que posea este estudiante. Considerando los coeficientes estandarizados, la Satisfacción del estudiante en su proceso de enseñanzaaprendizaje influye más significativamente que las otras 2 variables.

La Calidad del Proceso Enseñanza-Aprendizaje influye significativamente en la fidelidad del estudiante (Cantero y Mato, 2014; Candelas et al., 2013; Pereira, 2011; Manzano, 2010; Agustín y Domelis, 2009; Donoso y Schiefelbein, 2007). Además se puede indicar que ejerce una influencia indirecta en la Fidelidad a través de la Satisfacción del estudiante (Jiménez et al., 2011; Álvarez et al., 2014; Salinas et al., 2008). La satisfacción del estudiante, influye de manera positiva y significativa sobre la fidelidad del estudiante (Schlesinger et al., 2014; Candelas et al., 2013; Gómez et al., 2013; Suárez, 2012; de la Fuente et al., 2010), por lo que la fidelidad del estudiante y consecuentemente las recomendaciones que puede realizar y por lo tanto, la captación de nuevos estudiantes se ve influenciado por la satisfacción con respecto a los resultados y experiencias asociadas con su educación, logrando así consolidar la relación con su CES.

La red de apoyo externo es una variable que impacta directa e indirectamente y de manera significativa en la Fidelidad del estudiante. La relación directa es consistente con lo expresado en otras investigaciones (Lilian, 2014; de Val Merino, 2013; Barroso y Colón, 2009; Renteria y Andreade, 2007; Bertoglia, 2005). Además esta relación es menos influyente que la Calidad del Proceso Enseñanza-Aprendizaje, según los coeficientes estandarizados. La influencia indirecta es a través de la Satisfacción del estudiante, ratificando investigaciones previas (Martínez et al., 2014; Blazquez et al., 2013; Santana y Feliciano, 2011; Bernasconi, 2009; Cabrera et al., 2006), en donde el apoyo de sus compañeros de carrera, amigos, familiares o empresarios impactan en la satisfacción del estudiante dado que los aspectos académicos como los sociales son dimensiones influyentes.

Por su parte, la red de apoyo externo influye de manera positiva y significativa sobre la calidad del proceso enseñanza-aprendizaje, resultados que están en la línea expuesta por otras investigaciones (Cantero y Mato, 2014; Avagliano y Vega, 2013; Conchado y Carot, 2013; Montoya y Aguilar, 2013; López et al., 2012; Suarez, 2012; Aguiar et al., 2010; Saraiva, 2008). Además los resultados demuestran que si bien ambas variables influyen en la Satisfacción del estudiante de pregrado, la Calidad del Proceso de EnseñanzaAprendizaje tiene una mayor influencia que la Red Externa. 
Las estrategias centradas en mejorar la satisfacción del estudiante y su fidelidad se deben convertir en decisiones estratégicas en los CES. Estas correlaciones representan un paradigma de gestión educacional que es prioritario en Chile, dado los actuales desafíos de la educación superior, donde la calidad de la educación es simultáneamente una meta de corto plazo como también un objetivo estratégico, que debe impactar en las decisiones de los gestores educacionales, consistente con lo expresado por Pedraja y Rodríguez (2015); Candelas et al. (2013); Jiménez et al. (2011); de la Fuente et al. (2010). En este escenario se hace necesario estimular que el estudiante también asuma un rol de "cliente-estudiante", para quien la satisfacción y participación en los procesos de enseñanza-aprendizaje es imprescindible, lo que es consistente (Negrao et al., 2014). Complementariamente, este tipo de estudiantes dado su empoderamiento y rol activo debe ir generando paulatinamente un mayor nivel de fidelidad con su organización (en esta investigación los valores son de $63,2 \%$ y $68,8 \%$, a nivel de la Universidad y la carrera, respectivamente).

Por lo tanto, en los CES se debe asumir como una práctica cotidiana la innovación en gestión y marketing educacional, a nivel de los estudiantes de pregrado. La "rentabilidad" de las inversiones asociadas, además se verá reflejado en las contribuciones y calidad de la relación con los egresados, quienes son participantes relevantes de la red de apoyo externo para mejorar la satisfacción y el proceso de enseñanza-aprendizaje y por ende también es clave en el desarrollo sustentable de la Universidad, evidencia que está alineado a lo expresado por Martínez et al. (2014); Schlesinger et al. (2014); Blázquez et al. (2013); Santana y Feliciano (2011).

En el CES estudiado, el proceso educativo genera un nivel de satisfacción medio, cuyos valores son del $64,3 \%$ y $69,8 \%$, con la Universidad y la carrera, respectivamente. Se evidencia una brecha de insatisfacción necesaria de reducir. Esto representa un desafío para continuar mejorando la calidad y efectividad de los procesos internos, así como también un impulso para repotenciar la implementación de estrategias y acciones, que sean factibles técnica, económica y culturalmente, para integrar como un socio clave a las redes de apoyo externos. Particularmente, los egresados del mismo CES, junto con los estudiantes de pregrado, deben ser empoderados para contribuir en el ajuste, optimización y reinvención de todo proceso que fortalezca el posicionamiento de la Universidad y la carrera en la cual estudia y/o se tituló. Lo que además instala como desafío formar un estudiante con capacidades intraemprendedoras como se requiere en otras organizaciones de otros sectores de la economía, pues esto permitirá que la organización se renueve desde adentro, asumiendo un rol activo, en sintonía con lo expresado por Rubio (2015) y Moriano et al. (2009).

Si bien los resultados de esta investigación no se pueden generalizar a otras Universidades de Chile, tampoco se puede negar que otras investigaciones nacionales e internacionales, posicionan al estudiante como un actor central, aun sin explicitarlo, asignándole el rol de estudiante-cliente. Por ello, es necesario que estas organizaciones de educación lo integren planificadamente en la co-creación de innovaciones en procesos de apoyo y en los servicios educacionales. Por ello, en futuras investigaciones un tema relevante es integrar el "design thinking" como una filosofía de gestión permanente y no como una moda en los CES, como una herramienta útil en la gestión y desarrollo del estudiante-cliente y desde allí en el logro de su fidelización y contribución en el liderazgo competitivo de su universidad. Tal como lo expresan Hoegg et al. (2010) y Vergantini (2009); las organizaciones están viendo a la orientación en diseño como una oportunidad de diferenciación y últimamente como una fuente de ventaja competitiva y éxito en el mercado.

Al validarse con confiabilidad estadística la correlación de 3 variables con la fidelización del estudiante de pregrado, considerando la relevancia que el estado del arte le asigna a esta variable dependiente, mismas que están presentes en los 3 ejes del modelo educativo del CES estudiado (el estudiante es el centro de su quehacer formativo, se asume la formación integral de los estudiante y éste además es el Agente responsable y activo de su propia formación), es inevitable establecer que la gestión educacional en esta Universidad y en otros con su perfil a nivel nacional, deberán a lo menos iniciar o instalar reingenierías organizacionales, sea a nivel de los estilos de liderazgos, mecanismos de toma de decisión y arquitectura organizacional, para lograr de forma efectiva que sus procesos de dirección estratégica y modelos educativos, ampliamente difundidos, generen en el corto y largo plazo los resultados esperados por el estudiante, la Universidad, el mercado laboral y la sociedad, lo que está alineado con otras investigaciones (Avagliano y Vega, 2013; Ojeda, 2013).

\section{CONCLUSIONES}

Con base en el estudio realizado y presentado en este artículo, se pueden extraer las siguientes conclusiones principales:

1) La fidelización de un estudiante de pregrado requiere de una gestión proactiva, sistémica de variables que son interdependientes entre sí, como la Satisfacción del estudiante, Calidad del Proceso de Enseñanza- 
Aprendizaje y Red de Apoyo Externo, lo que implica, dar relevancia tanto a la gestión del entorno y de los activos claves; como a los estudiantes y los procesos que producen su transformación y desarrollo.

2) No se puede tener estudiantes fidelizados con su Universidad y por ende con su carrera, si éste no está satisfecho con el proceso de enseñanza-aprendizaje.

3) En universidades equivalentes del concierto latinoamericano, es un desafío que en la gestión educacional se instale reingenierías organizacionales alineadas a sus modelos educativos, tal que, el estudiante asuma el rol de estudiante-cliente.

\section{AGRADECIMIENTOS}

Los autores agradecen el apoyo entregado por la Universidad de La Serena para terminar exitosamente esta investigación.

\section{REFERENCIAS}

Aguiar, G., J. Peinado, J.C. Cunha y B. Aguiar, Las visitas Técnicas a Empresas como parte del Proceso de Enseñanza-Aprendizaje en Ingeniería Mecánica, Formación Universitaria, 3(5), (2010)

Agustín, M., y M. Domelis, Desarrollo de un Instrumento para Medir la Satisfacción Estudiantil en Educación Superior, Docencia Universitaria, 10(2), (2009)

Álvarez, J., E. Chaparro y D. Reyes, Estudio de la Satisfacción de los Estudiantes con los Servicios Educativos brindados por Instituciones de Educación Superior del Valle de Toluca, Revista Iberoamericana sobre Calidad, Eficacia y Cambio en Educación, 13(2), 5-26, (2014)

Asún, R., C. Zúñiga, y M. Ayala, La formación por competencias y los estudiantes: Confluencias y divergencias en la construcción del docente ideal, Calidad en la educación, 277-304, (2013)

Avagliano, A.R. y S.A. Vega, Mejora del proceso de Enseñanza y Aprendizaje en la Carrera de Ingeniería de Ejecución Mecánica. Diseño Micro-Curricular Basado en Resultados de Aprendizaje, Formación Universitaria, 6(4), 3-12, (2013)

Barroso, R. y H.M. Colón, Redes de apoyo a la formación universitaria a distancia en la UNAM, una propuesta para contribuir al derecho a la educación superior. México D.F., (2009)

Bernasconi, G., Red AGE: La red de apoyo a la gestión educativa en Ibero-América, Montevideo, (2009)

Bertoglia, L., La interacción profesor-alumno, una visión desde los procesos atribucionales, Psico Perspectivas, 4, 57-73, (2005)

Bitar, S., Tendencias mundiales y el futuro de la educación superior en Chile, Senior Fellow Inter-American Dialogue, http://www.bitar.cl/wp-content/uploads/2015/05/bitar-tendencias-mundiales-pdf-final.pdf (2015).

Blázquez, J.J., J. Chamizo, E.I. Cano y S. Gutiérrez, Calidad de vida universitaria: Identificación de los principales indicadores de satisfacción estudiantil, Revista de Educación, 0(362), (2013)

Bollen, K.A., Structural equations with latent variables, New York: John Wiley \& Sons, (1989)

Cabrera, L., J. Tomás, P. Álvarez y M. González, El problema del abandono de los estudios universitarios, RELIEVE, 12(2), 171-203, (2006)

Candelas, C., M. Gurruchaga, A. Mejías y L. Flores, Medición de la satisfacción estudiantil universitaria: Un estudio de caso en una institución Mexicana, Revista Iberoamericana de Ingeniería Industrial, 5(9), 261-274, (2013)

Cantero, J., y M. Mato, El proyecto docente en la universidad española según el Espacio Europeo de Educación Superior, Calidad en la educación, (40), (2014)

Chin, W.W., The Partial Least Squares Approach to Structural Equation Modeling, in G.A. Marcoulides [ed.], 295-336, Modern Methods for Business Research, Mahwah, NJ: Lawrence Erlbaum Associates, (1998)

Conchado, A., y J. Carot, Puntos fuertes y débiles en la formación por competencias según, Revista de Docencia Universitaria, 11(1), 429-446, (2013)

de la Fuente, H, M. Marzo y M. Reyes, Análisis de la satisfacción universitaria en la facultad de ingeniería de la universidad de Talca, Ingeniare. Revista chilena de ingeniería, 18(3), 350-363, (2010) 
del Val Merino, C., La integración del alumno al mundo laboral. El paradigma de la Inteligencia Emocional en el marco del Espacio Europeo de Educación Superior (EEES), Estudios sobre el Mensaje Periodístico, (19), 129-138, (2013)

Donoso, S. y E. Schiefelbein, Análisis de los modelos explicativos de retención de estudiantes en la universidad: una visión desde la desigualdad social, Estudios Pedagógicos, 33(1), 7-27, (2007)

Espejel, J., D. Camarena y S. Sandoval, Alimentos tradicionales en sonora, México: factores que influyen en su consumo, Innovar, 24(53), 127-139, (2013)

Falk, R. y N. Miller, A primer for soft modeling, 1ra edition, Akron, O H: University of Akron Press, (1992)

Fornell, C. y D.F. Larcker, Evaluating Structural Equation Models with Unobservable Variables and Measurement Error, Journal of Marketing Research, 18(1), 39-50, (1981)

Fuentes, M. y B. Moliner, Antecedentes del Éxito de la Relación entre Restaurantes y sus Clientes, Revista Innovar, 24 (53), 99-112. (2014)

Gaxiola, J.C., S. González y Z. Contreras, Influencia de la resiliencia, metas y contexto social en el rendimiento académico de bachilleres, Revista Electrónica Investigación Educativa, 14(1), 164-181, (2012)

Gefen, D. y D. W. Straub, A Practical Guide to Factorial Validity Using PLS-Graph: Tutorial and Annotated Example, Communications of the AIS, 16(5), 91-109, (2005)

Gómez, D., E. Martínez, R. Eugenia, R. Recio y H. López, Lealtad, Satisfacción y Rendimiento Académico en los Estudiantes de la UASLP-UAMZM, Sophia, 9, 1-17, (2013)

González, M. y E. Backhoff, Validación de un cuestionario de contexto para evaluar sistemas educactivos con modelos de ecuaciones estructurales, E-Journal of Educational Research, Assessment and Evaluation, 16(2), 1-17, (2010)

Hoegg, J., W. Alba y W. Dahl, The good, the bad, and the ugly: Influence of aesthetics on product feature judgments, Journal of Consumer Psychology, 20(4), 419-430, (2010)

Jiménez, A., B. Terriquez y F. Robles, Evaluación de la satisfacción académica de los estudiantes de la Universidad Autónoma de Nayarit, Revista Fuente, (2011)

Lilian, H. H., Graduate Student's Perspectives on Effective Teaching, 25(2), (2014)

López, R., N. Pérez y G. López, El pizarrón, la influencia de su uso en la calidad del proceso de enseñanza aprendizaje, Revista Educación Médica del Centro, EDUMECENTRO, 4(3), 206-215, (2012)

Manzano, V., Universidad comprometida, Polis, Revista de Universidad Bolivariana, 9(27), 553-558, (2010)

Martínez, Z., M.F. Páramo, M.A. Guisande, C. Tinajero, L. da Silva y M.S. Rodríguez, Apoyo social en universitarios españoles de primer año: propiedades psicométricas de Social Support Questionnarie-Short Form y el Social Provisions Scale, Revista Latinoamericana de Psicología, 46(2), 102-110, (2014)

Mercado, A., y P. Gil, Caracteristicas psicométricas del "Cuestionario para la evaluación del Síndrome de quemarse por el trabajo" en maestros mexicanos, Revista de Educación, (2012)

Montoya, F. y J. Aguilar, The Linkage of University-Industry within Business Internships: A Conceptual Model Using Idea Generation Techniques, Journal of Technology Management \& Innovation, 8(1), (2013)

Moriano J., G. Topa, E. Valero y J. Levy, Identificación organizacional y conducta intraemprendedora, Anales de Psicología, 25(2), 277-287, (2009)

Negrao, R., J. Amado, M. Carneiro y A. Mazzo, Satisfacción de los estudiantes con las experiencias clínicas simuladas: validación de escala de evaluación, Revista Latino-Americana de Enfermagem, 22(5), (2014)

Ojeda, M., La planificación estratégica en las instituciones de educación superior mexicanas: de la retórica a la práctica, CPU-e, Revista de Investigación Educativa, 0(16), 110-118, (2013)

Patiño, H., Educación humanista en la universidad, Perfiles Educativos, 23-41, (2012)

Pedraja, L. y E. Rodríguez, El aseguramiento de la calidad: un imperativo estratégico en la educación universitaria. Ingeniare, Revista Chilena de Ingeniería, 23(1), 4-5, (2015)

Pereira, M., Nuevas tendencias en la evaluación de la calidad de las universidades: los índices de calidad percibida y satisfacción de los egresados (con modelos de ecuaciones estructurales), 39(3), 73-84, (2011) 
Renteria, E. y V. Andrade, Representaciones y acciones de los alumnos universitarios frente a la empleabilidad, Issue, (20), 130-155, (2007)

Rodríguez, E., La educación superior en Chile y el rol del mercado: ¿culpable o inocente?, Ingeniare. Revista chilena de ingeniería, 20(1), 126-135, (2012)

Rubio, G., Las contribuciones del intraemprendimiento a la estrategia de manufactura, Revista Dimensión Empresarial, 13(1), 95-109, (2015)

Salinas, A., J. Morales, y P. Martíntez, Satisfacción del estudiante y calidad universitaria: Un análisis explicatorio en la unidad académica multidisciplinaria agronomia y ciencias de la universidad autónoma de Tamaulipas, México, Revista de Enseñanza Universitaria, (31), 39-55, (2008)

Sanguino, R.G., La competitividad de la administración local. Modernización a través de la gestión del conocimiento, Primera edición edición, Madrid, (2006)

Santana, L. y L. Feliciano, Percepción de apoyo de padres y profesores, autoconcepto y toma de decisiones en bachillerato, Revista de Educación, 493-519, (2011)

Saraiva, M., La calidad y los "clientes" de la enseñanza superior portuguesa, Horizontes Educacionales, 13(2), 41-54, (2008)

Schlesinger, W., A. Cervera y H. Calderón, El papel de la confianza, la imagen y los valores compartidos en la creación de valor y lealtad: aplicación a la relación egresado-universidad, Revista Española de Investigación de Marketing ESIC, 18, 126-139, (2014)

Suárez, M., Los estudiantes como consumidores. Acercamiento a la mercantilización de la educación superior a través de las respuestas a la Encuesta Nacional de Alumnos de Educación Superior (ENAES), Perfiles educativos, 35(139), (2012)

Vergantini, R., Design-driven innovation: Changing the rules of competition by radically innovating what things mean, Harvard Business Press, (2009)

Vinzi, V., W. Chin, J. Henseler y H. Wang, Handbook of Partial Least Squares: Concepts, Methods and Applications 1st edition, Berlin: Springer-Verlag Berlin Heidelberg, (2010)

Williams, B., T. Brown, y A. Onsman, Exploratory factor analysis: A five-step guide for novices, Australasian Journal of Paramedicine, 8(3), (2010) 